
\title{
25 Research Suare \\ Phylogenetic and Ultrastructural Characterization of Bed Bugs in the Southwest of Iran
}

\author{
Somayeh Bahrami ( $\nabla$ s.bahrami@scu.ac.ir) \\ Shahid Chamran University of Ahvaz \\ Ismaeil Alizadeh \\ Kerman University of Medical Sciences \\ Fatemeh Pazhoom \\ Shahid Chamran University of Ahvaz \\ Susan Cork \\ University of Calgary \\ Chukwunonso O.Nzelu \\ University of Calgary \\ Ali Reza Alborzi \\ Shahid Chamran University of Ahvaz
}

\section{Research}

Keywords: Bed bug, Cimex hemipterus, Iran, Phylogenetic tree, Scanning electron microscopy

Posted Date: November 24th, 2020

DOI: https://doi.org/10.21203/rs.3.rs-112151/v1

License: (c) (i) This work is licensed under a Creative Commons Attribution 4.0 International License. Read Full License 


\section{Abstract}

Background: Bed bugs belong systematically to Order Hemiptera; Suborder Heteroptera; Family Cimicidae are of public health importance as ectoparasites of mammals and birds, however, only a few species are the putative ectoparasites of humans. Bed bugs are a wingless bloodsucking hemipterous bug (Cimex spp.) sometimes infesting houses and especially beds and feeding on human blood. Correct species identification is very important in order to design targeted strategies for surveillance and control of bed bugs in a given area.

Methods: Adult bed bugs were collected from houses located in the southwest of Iran. The specimens were morphologically identified to the species level and then confirmed using molecular methods.

Results: The mtDNA 16S rRNA sequences obtained from the specimens, and phylogenetic tree derived, showed that all the sequences belong to Cimex hemipterus. The Disparity Index among results showed that all the specimens were of a heterogeneous population. To the best of our knowledge, the leg structure of this species has not previously been documented and this is the first report of an open-closed rack system in the legs of $C$. hemipterus.

Conclusions: Previous studies claimed that $C$. lectularius is the abundant species of Cimicids in Iran. But this investigation showed that $C$. hemipterus is the prevalent species in the southwest of Iran.

\section{Background}

Cimex is a genus of insects in the family Cimicidae which are hematophagous ectoparasites that normally feed on the blood of birds and mammals. Cimex lectularius, $C$. hemipterus, and Leptocimex boueti are three main species considered as ectoparasites of humans [1,2]. These nocturnal bloodsucking ectoparasites cause itching, anxiety, insomnia, sleep deprivation, emotional stress, secondary infections, and may even lead to anemia and iron inadequacies $[3,4]$. Also, bed bugs can cause economic difficulty due to the cost of extermination and the need to replace infested furniture [5]. Furthermore, they represent an important public health threat that may affect the housing industry, the tourism industry, and public institutions. The application of insecticides is the primary tactic to control bed bugs [6]. However, lately, resistant bed bugs have re-emerged in large numbers in many parts of the world partly due to the extensive and repeated as well as incorrect usage of insecticides [7]. Passive dispersal is the main way for cimicids to reach new hosts. Bed bugs can be transported by humans in clothing and stuff $[6,8]$, and they have been detected on people traveling by trains, ships, airplanes, and cars. Moreover, humans, birds, and bats can also be hosts for bed bugs, and they are considered to be crucial in the distribution of cimicids [9]. Global warming and limited awareness about the biology of bed bugs are other explanations for the recent recurrence of these parasites. Knowledge of the genetic variation within medically important insect species is an important factor required for understanding disease epidemiology, vector transmission, and disease control [10]. Although implicated, bed bugs are not commonly considered to transmit infectious diseases. However, mechanical transmission of infectious particles of kala-azar, typhus, anthrax, plague, tularemia, relapsing fever, Q-fever, hepatitis B virus, and human immune deficiency virus has formerly been recorded $[1,11,12]$. Conducting genetic studies on bed bugs can give information to help better understand their distribution patterns and population structure, and may also provide insight into their movement for medical purposes [13]. To date, very few studies have been performed on the genetic variation of bed bugs by using either molecular or biochemical methods in Iran. Therefore, the objective of this study was to determine the predominant species of bed bugs in Iran. Furthermore, phylogenetic analysis and the detailed morphological characteristics of the collected bugs were studied using a scanning electron microscopy (SEM).

\section{Methods}




\section{Bed bug (Cimex spp.) collection and rearing}

The specimens were collected from March to November 2019 in Ahvaz, Khuzestan province (southwest of Iran). Ahvaz is divided into five public health regions (i.e., Northern, Southern, Eastern, Western, and Central). The specimens were collected from the houses in which their infestation to bed bugs was reported to the pest control companies and health centers in five regions. For inspection crevices, cracks, and mattresses were checked. Bugs were caught using an aspirator and bed bug trap. A total of 95 bugs were caught from 23 houses located in five regions. The bugs were reared in plastic rearing containers covered with fine mesh chiffon cloth and transferred to the Department of Parasitology, Faculty of Veterinary Medicine, Shahid Chamran University of Ahvaz.

\section{Ethical consideration}

This study received ethical approval from the Ethics Committee of the Shahid Chamran University of Ahvaz (No. EE/99.24.3.70110/SCU.AC.IR). All the IDI participants provided written informed consent. The results of this study have been presented to the Department of Parasitology, Faculty of Veterinary Medicine, Shahid Chamran University of Ahvaz, Ahvaz, Iran.

\section{Molecular examination}

\section{DNA extraction and PCR amplification}

DNA was extracted from 20-bed bugs using a genomic DNA purification kit (SinaClon Bioscience, Karaj, Iran) according to the manufacturer instructions and $50 \mathrm{ng}$ of the extract was used as PCR templates. The insect mitochondrial 16S rRNA gene of the 428-bp fragment was amplified using the primers LR-J-13007 (5'-

TTACGCTGTTATCCCTAA-3') [14] and LR-N-13398 (5'-CGCCTGTTTATCAAAAAC-3') [15]. The reaction was carried out in a volume of $25 \mu \mathrm{L}$ using $12.5 \mu \mathrm{L}$ Taq DNA Polymerase Master Mix Red (Amplicon, Odense, Denmark) and a pair of primers ( $1 \mu \mathrm{M}$ each). Each PCR reaction comprised a negative control, consisting of the reaction mix and $2 \mu \mathrm{L}$ of DNase/RNase-free water instead of DNA. After an initial denaturation at $94^{\circ} \mathrm{C}$ for $2 \mathrm{~min}, \mathrm{PCR}$ amplification was repeated with 35 cycles consisting of denaturation at $94^{\circ} \mathrm{C}$ for $45 \mathrm{~s}$, annealing at $46^{\circ} \mathrm{C}$ for $45 \mathrm{~s}$, and extension at $72^{\circ} \mathrm{C}$ for $60 \mathrm{~s}$. This was followed by a final extension at $72^{\circ} \mathrm{C}$ for $5 \mathrm{~min}$. PCR products were electrophoresed in $1.5 \%$ agarose (SinaClon Bioscience) in Tris-acetate-EDTA (TAE) buffer, stained with Green Safe stain (SinaClon Bioscience), and visualized under ultraviolet light. Since the city is divided into five public health regions one sample from each region was sequenced. Amplicons corresponding to the expected size (428 bp) were purified using a PCR purification kit (Vivantis, Revongen Corporation Center, 47600 Subang Jaya, Selangor Darul Ehsan, Malaysia). Amplicons were sequenced using specific primers and a Big Dye Terminator V.3.1 Cycle Sequencing kit in an ABI 3130 Genetic Analyzer (Applied Biosystems, Foster City, California 94404, USA).

\section{Sequence variation and phylogenetic tree construction}

The nucleotide sequences of five samples from various regions were aligned for variation positions. Multiple sequence alignment analysis was performed using nBLAST (http://blast.ncbi.nlm. nih.gov/Blast.cgi) and ClustalW2 software (Conway Institute UCD, Belfield, Dublin, Ireland). The phylogenetic tree was constructed by the Maximumlikelihood method using the Hasegawa-Kishino-Yano model with gamma-distributed rates among sites. The reliability of an inferred tree was tested by 1000 bootstrap. Pediculus humanus capitis accession no. AY139928.1 was considered as an outgroup. Evolutionary analyses were conducted in MEGA X. DnaSP [16] software was used to determine the haplotype diversity $(\mathrm{Hd})$, the polymorphic site, the average number of nucleotide differences [17], nucleotide diversity (ð), singleton variable sites, the parsimony-informative sites, and genetic differentiation (Fst) [18]. 


\section{Morphological examination and scanning electron microscopy preparation}

Bugs were killed by benzene vapor and immediately placed in 70\% ethanol. Any extracellular debris, such as the blood, mucus, other body fluids, and tissue fragments were removed carefully. The specimens were photographed in a standardized way using stereoscopic microscopy (Olympus SZX9) and a digital camera (Olympus C-5060, Olympus, Tokyo, Japan) operated by Photo Micro 2.0. The measurements were taken using MeasurelT (Olympus). The bugs were identified using taxonomic keys [19]. For scanning electron microscopy samples were fixed in buffered $2 \%$ glutaraldehyde $(\mathrm{pH}$ 7.2) for $12 \mathrm{~h}$. The fixed bugs were washed in phosphate buffer $(\mathrm{pH} 7.2)$ for thrice and then in double distilled water followed by acetone dehydration. After acetone dehydration, the specimens were dried in a critical point drier using liquid $\mathrm{CO}_{2}$. Samples were then attached in aluminum stubby adhesive tapes. Cimex spp. were then gold coated in a sputter coater (Polaron-SC7620) and were examined under SEM (LEO 1455 VP, operated at $30 \mathrm{kV})$.

\section{Results}

\section{Sequence comparison and phylogeny of bugs}

The average size fragment of the gene from the amplified bugs was $333 \mathrm{bp}$. The amplified gene sequences varied from 360 to $388 \mathrm{bp}$. The consensus gene sequences were blasted in the NCBI Genbank database, and they showed a high degree of homology with the 16S ribosomal RNA gene of C. hemipterus in GenBank at 88.38-90.33\% identity. The nucleotide sequences of the 16S rRNA gene from the $C$. hemipterus are available in the GenBank databases (http://www.ncbi.nlm.nih.gov/genbank/) under the accession numbers MT520974- MT520978. After few processes of removal and alignment of sequences using the T- coffee molecular software, $323 \mathrm{bp}$ of the partial $C$. hemipterus of the 26 sequences of Cimex sp. and one outgroup, $P$. humanus were successfully obtained. The overall frequency distributions of nucleotides in the first, second, and third codon positions are as follows: $\mathrm{T}=40.7 \%, 40.3 \%$, and $43.2 \%$; $C=7.5 \%, 13.2 \%$, and $10.3 \% ; A=37.5 \%, 28.9 \%$, and $30 \% ; G=14.3 \%, 17.6 \%$, and $16.5 \%$, respectively. Analysis of the sequences revealed a mean GC content of $26.5 \%$. The analysis of the homogeneity of the substitution patterns between sequences using the Disparity Index Test (Monte Carlo Test) with 500 replicates reveals no significant differences across the sequences (Table 1). For estimation of evolutionary divergence between sequences, pairwise distances were computed, and the results are shown in Table 2.

The sequence analysis detected 28 polymorphic sites (16 singletons and 14 parsimony informative sites) among sequences. Results of DNA polymorphism analysis of the five isolates sequences showed that the nucleotide diversity (ð) was 0.57 . The total number of mutations (Eta) was 30 while the number of haplotypes (h) was five. The haplotype diversity $(\mathrm{Hd})$ was 1 , with a standard deviation of 0.02 . The average number of nucleotide differences per site between two sequences $(\mathrm{Pi})$ and $\mathrm{G}+\mathrm{C}$ content at non-coding positions was 0.04 and 0.26 , respectively. Based on the Neutrality test results non-significant Tajimo's D and Fu's Fs values were obtained. Genetic differentiation among the population was 0.6. Basic parameters of genetic diversity and neutrality tests are shown in Table 3 . Three genomic conserved regions were detected along the 323-bp region (Table 4).

In the phylogenetic tree, the 27 sequences were classified into four large clades (Fig. 1), and the phylogenetic analysis showed that Iranian bugs are grouped with the $C$. hemipterus. The constructed phylogenetic tree could clearly separate two major clades of $C$. hemipterus and $C$. lectularius, although they belonged to the same genus. Interestingly, the tree showed that Iranian specimens were clearly grouped closely together in one branch with a $95 \%$ 
bootstrap support value (Fig. 1). The obtained pattern confirms the validity of 16S rRNA analysis to elucidate the minor differences among closely related species.

\section{Morphology of bed bugs (C. hemipterus)}

In this study, adult bed bugs were oval and mahogany-colored, and they ranged in length from about 4.6 to $5 \mathrm{~mm}$, with abdomens 2.6 to $2.8 \mathrm{~mm}$ wide. The heads were small and cylindrical, with two knoblike multifaceted eyes. Ocelli were absent but compound eyes were well developed (Fig. 2). As Fig. 2 shows, the antennae were four segmented (scape, pedicel, flagellum, and distal flagellum) and placed between the clypeus and eye. The shortest segment was the first segment (scape), and the third and fourth segments were more transparent, slender, and flat than the first and second segments. From the middle of the fourth segment, a deep groove was seen (Fig. 2).

By carefully examining sensilla external shapes and dimensions on the antenna, we could distinguish two types of chaetic, one type of basiconic and one type of coeloconic sensilla in females and males.

Sensilla chaetica type 1 (ch1) were very long, sickle-shaped bristles with longitudinal grooves and blunt tips. The base of the bristles rests on the distal edge of a wide flexible socket and projected from the antennal surface at an angle of $19-96^{\circ}$ (mean value of $57.5^{\circ}$ ) (Fig. 2). The ch1 had the longest pegs, with a mean length of $97.1 \mu$ m. Sensilla chaetica type 2 (ch2) were more or less straight bristles, 65-96 $\mu \mathrm{m}$ long, with a hair shaft that strongly tapers from about half of its length and ends in a sharp tip (Fig. 2). The tip was often bent away from the antennal surface. The base of the hair shaft was inserted at an angle of $47^{\circ} \pm 6.5^{\circ}$ (mean \pm std.dev.) into a very tight socket and rested on its concave surface. The surface structure was similar to that of ch1 (i.e., longitudinal grooves present). Chaetica sensilla were the most prominent and longest sensilla on the antennal surface.

Sensilla basiconica (ba) were primarily slightly curved, cone-like hairs, smooth-walled without longitudinal grooves with numerous pores on the cuticular surface, and ended to the blunted tips. The hair shaft inserted basally at an inclination angle that ranged from $19^{\circ}$ to $40^{\circ}$ into a socket with a diameter of 2.9-6.4 $\mu \mathrm{m}$. At its base, the hair shaft had a diameter that ranged from 2.2 to $3.4 \mu \mathrm{m}$. Sensilla basiconica were found mainly on both the flagellar segments (Fig. 2).

Sensilla coeloconica (co) were peg-in-pit sensilla, consisting of a small peg set on the floor of a chamber sunken into the cuticle. A small central aperture with a diameter of 2.9-6.5 $\mu \mathrm{m}$ connected the peg inside the chamber with the surrounding air (Fig. 2). Based on the distribution of the various types of sensilla, no sexual dimorphism could be noticed in $C$. hemipterus.

Mouthparts formed a pointed beak or rostrum that folded under the head in a ventral groove up to the first pair of legs. The mouthparts were modified for piercing the skin and sucking the blood. They consisted of a labrum, a pair of mandibles, and a pair of maxillae. The labrum was short, and it covered the mid-dorsal groove of the rostrum. The labium formed an elongated incomplete hollow tube or sheath called the rostrum. The labium was three-segmented and consists of two lateral lobes and a middle lobe (apical plate) situated on the ventral side. There were sensory structures on the tips of the lateral lobes. The labium was mid dorsally grooved to enclose the four needle-shaped stylets, two mandibles, and two maxillae (Fig. 3). The maxillary and labial palps were absent. Different studies showed that there are a number of differently shaped and differently sized chemo- (gustatory, contact chemoreceptors) and mechanosensilla on the tip of the labium of true bug. In this paper, we are not discussing these sensilla anymore.

The thorax consisted of a narrow canoe-shaped pronotum, a mesonotum (scutellum) which was covered dorsolaterally by reduced fore wings called hemelytral pads, and a mesonotum (scutellum) hidden below the latter 
(Fig. 3). The prothorax was broad, receiving the head in its concave anterior margin, with the sides extending wing-like laterally (Fig. 4). The same as what Usinger (1966) explained about $C$. lectularius we found that in the ventral side of C. hemipterus the widely separated sternal apophyseal pits lied between and were concealed by the coxae [19]. The sclerotized area between the pits and partially surrounding them was certainly sternal. The trochantin appeared as a small, detached, sclerite in the membrane anterior to the coxae. The mesothoracic spiracle lied in a small plate just posterior to the coxae. On the ventral side, the mesothorax was almost completely sclerotized, only slight areas about the coxal foramina remaining membranous. At the margin of the body a narrow, sclerotized isthmus connected the sclerotization of the mesothorax and metathorax. In this isthmus, the spiracles of the metathorax lied close to the coxal condyle of the mesothorax. Widely separated between the coxae were the sternal apophyseal pits, which were borne within narrow sclerotized arms branching from the median sclerotized area.

The metathorax was in general similar to the mesothorax in the arrangement of its ventral sclerotization, but the encroachment of the coxae of the mesothoracic legs has narrowed its lateral positions.

All the parts of the legs were always present except pulvilli and arolia. On the ventral surface of each leg, a tuft of setae was seen on a pad-like extension at the apex of the tibia. The tibia brush varied in length from $35 \mu \mathrm{m}$ to $60 \mu \mathrm{m}$. The pad-like extension at the apex of the tibia had a very wrinkled surface which may be related to glandular openings. In the present study, we found that trichoid sensilla were disseminated among the tibial brush setae.

There were three tarsal subsegments (or tarsomeres) and paired pretarsal claws on each of the six legs. The terminal segment was about as long as the other 2 together. Little is known about the ultrastructure of $C$. hemipterus legs. Figure 4 shows several structures in pretarsus and as seen in Fig. 4 open-closed system was found in C. hemipterus legs. The open-closed system was formed by the central rack system (crs), two lateral telescopic columns (Itc), and finger-like processes with possible sensory function (flp) (Fig. 4). The rack system could be a system to lock the position of the claw, as was the pad-like structure and always two spiniform setae were in its base.

The abdomen was 10 segmented. The 1 st and 2 nd abdominal segments were fused. In adult male bug, the abdomen was narrower, and its tip was curved and slightly more pointed than in the female. The intersegmental membranes were wide and the second to fifth abdominal segments were membranous at the middle of the ventral surface, forming the so-called hunger folds. The primary genitalic structures of the female formed a recognizable homolog of the ovipositor and occupied the ventral aspects of the ninth and tenth segments.

Laterally, the paratergites of the eighth segment bore the last spiracles (Fig. 5). A pair of broad, flat plated called gonocoxae occupied the median two- thirds of the ventral aspect of the eighth segment. The lateral plates of the ninth segment were the fused paratergite and 2nd gonocoxa.

As shown in Fig. 5, a small incision was present on the right-handed posterior margin of the fifth sclerite. This incision is the opening of a copulatory pouch referred to as a spermalege (also known as paragental sinus or Berlese's organ). Spermalege is an organ specialized to receive hypodermically injected semen. The spermalege consisted of two parts, the ectospermalege, and endospermalege. The ectospermalege derived from the ectoderm and consists of a groove. The mesospermalege is derived from mesoderm and is a membrane-bound sac attached to the wall of the haemocoel, directly beneath the groove of the ectospermalege [20].

In male bugs, the intromittent organ is highly specialized for traumatic insemination. During traumatic insemination, a male bug inserts his intromittent organ into the ectospermalege and pierces the pleural membrane. The sperm is then injected into the mesospermalege, which contains hemocytes [20]. The spermatozoa then form a mass and diffuse through the wall of the mesospermalege, swimming through the hemocoel to a pair of seminal conceptacles, where 
sperm are stored before they migrate to the ovaries for fertilization. Seminal conceptacles have evolved independently of the bursa copulatrix and spermatheca [20].

The paramere arose close to its apex on the ventral side. The paramere was strongly curved to the left, its external portion lying in a furrow in the side of the ninth segment. The base of the paramere was retracted into the body for a short distance. The paramere was grooved on the outer or convex side, except apically where it was folded to form a tube. The aedeagus lied just above the base of the paramere (Fig. 5). The aedeagus was a long narrow tubular structure whose walls were not sclerotized. When not in use the aedeagus was telescoped into the capsule-like phallobase. It was probably extended by fluid pressure.

The entire abdomen was covered with numerous chaetae on both dorsal and ventral sides. At the tip of the abdomen of both the male and female bugs, there presented a tuft of relatively long chaetae. Based on electron microscopic results we found that in female bugs chaetae had three teeth while males were with four teeth in the tip (Fig. 5).

\section{Discussion}

The Fertile Crescent region of the Middle East, and areas along the Mediterranean Sea, have often been described by experts as the location of origin for the bed bugs (Cimex spp) [21]. One of the most populous countries in the Middle East is Iran. The identification of bed bugs in Iran has largely been based on insect morphology. Although this procedure can identify the adult stage easily, it is very difficult in immature stages or eggs [22]. Moreover, taxonomic identification requires considerable skill and taxonomic expertise and complete sample features. Nowadays, molecular techniques are available for the taxonomic identification of insects such as nucleotide sequence analysis, phylogenetic tree, and polymerase chain reaction-restriction fragment length polymorphism (PCR-RFLP) [23]. These techniques are fast, accurate, and highly sensitive; moreover, they can be performed for species identification for immature stages, cast skins, or incomplete specimens collected from the field.

Previous studies on the identification of bed bugs from Iran claimed that $C$. lectularius was the prevalent species in Iran [24-28]. But all these previous studies were based on morphological examination. While there is a significant similarity between the two species, there are minor morphological differences that may be hard to distinguish $[19,29]$. Furthermore, most of these studies were done in the northern and northwestern parts of Iran with attention to the various climates and geographical regions in Iran. In a recent study Hosseini-Chegeni et al. (2019), showed that the nymph and adult bug specimens collected from residential buildings in central and western parts of Iran were $C$. hemipterus based on the phylogenetic analysis of cytochrome c oxidase subunit 1 (COI) gene [30]. In the present study, samples were collected from the southwest of Iran. The results of sequences BLAST in the Genbank and phylogenetic tree showed that all the sequences obtained in this study belong to the $C$. hemipterus. Khuzestan province has an important impact on trade and commerce, tourism, and a steady influx of migrant workers. We think that the fauna of bed bugs in this province may be affected by climate changes, tourism industry, and international trades. The Disparity Index result showed that the specimens were of heterogeneous populations. The level of genetic differentiation indicated by FST was rated as FST $>0.25$ (huge differentiation), 0.15 to 0.25 (great differentiation), 0.05 to 0.15 (moderate differentiation) and FST < 0.05 (negligible differentiation) [31]. In this study, genetic differentiation among the population was 0.6 and indicated differentiation among the population.

Members of the Cimicidae show some common characteristics with other hematophagous arthropods and some unique features in the family. Insect antennae play a very important role in many crucial insect behaviors, such as mating, localization for oviposition, searching for food, aggregation, and escaping from dangers [32,33]. Antennae carry a wide range of sensilla types that serve a multiplicity of sensory modalities, including olfaction, gustation, 
mechanoreception, thermoreception, and hygroreception [34]. For $C$. hemipterus, location of its host, selection of a proper site on the body-part for sucking the blood and the act of taking in the blood meal, followed by an escape response on satiation, or on sensing danger, are all dependent on a rich complement of olfactory, gustatory, thermo-, hygro-, and mechanosensory sensilla [35]. Antennal sensilla from several dipterans' insects have been characterized [36-39]. Insect antennal sensilla usually occur in the form of hairs, pegs, and pits. Based on their morphological characteristics they can be termed as sensilla trichodea, placodea, coeloconica, basiconica, chaetica, Böhm's bristles, etc $[40,41]$. In this study, fine sensilla were found in each segment of the $C$. hemipterus antenna and the size and the distribution of sensilla were similar in both the sexes. Sensilla density was greater on the pedicel than the remaining segments. Results from Olson et al., (2014) antennectomy experiments showed that sensilla on the distal half of the pedicel are necessary for off-host aggregation by bed bugs [42]. In the present study, two types of chaetic, one type of basiconic and one type of coeloconic sensilla in females and males. Chaetica sensilla were the most prominent and longest sensilla on the antennal surface. Several studies indicate that cheatica sensilla are both chemoreceptors and mechanoreceptors [43-45]. They may protect the finer underlying structure on the antennae or perceive the difference in substrate texture and movement $[44,46]$. Sensilla basiconica was found mainly on both the flagellar segments (Fig. 2). Sensilla basiconica on the antennae are deduced to possess olfactory function [47]. Sensilla coeloconica was peg-in-pit sensilla, consisting of a small peg set on the floor of a chamber sunken into the cuticle. Wang et al. (2016) concluded that sensilla coeloconica type might be olfactory or humidity receptors, as demonstrated in other studies [48]. Based on the distribution of the various types of sensilla, no sexual dimorphism could be noticed in $C$.

hemipterus. A brief description of the bed bug tibial brush now usually referred to as the tibial pad [49] or the fossula spongiosa [18]. SEM analysis of Baker and Goddard (2018) revealed that near the apex of the tibial brush setae, they become distinctly spatulate with a smooth dorsal surface and a ventral surface with rounded protuberances [50]. Their transmission electron microscopy (TEM) analysis showed that the brush setae were hollow and had a porous cuticle. Different studies claimed that these setae are used for surface adhesion of insects. Furthermore, perhaps males use tibial brush setae to hold and position females during mating [50]. In the present study, we found that Trichoid sensilla were disseminated among the tibial brush setae. Seelinger and Tobin (1981) believed that the trichoid sensilla situated among the tibial brush setae were likely involved in perceiving sensory cues from various substrates [51]. In the general explanation of the insect legs, the tarsal apex bears the structures which form the pretarsus, the terminal segment of the legs [52]. In this study, we showed several structures in pretarsus and as seen in Fig. 4 open-closed system was found in $C$. hemipterus legs. The open-closed rack system could be a system to lock the position of the claw, as was the pad-like structure and always two spiniform setae were in its base. The openclosed system has been seen in sucking lice (Anoplura), P. humanus, and Haematopinus apri [53]. To the best of our knowledge, this is the first report of an open-closed rack system in the legs of $C$. hemipterus.

\section{Conclusions}

Previous studies claimed that $C$. lectularius is the abundant species of Cimicids in Iran. But, in this investigation, the results of sequences BLAST in the Genbank and phylogenetic tree showed that all the sequences belong to the $C$. hemipterus and all the samples were of heterogeneous populations. Furthermore, the mtDNA 16S rRNA gene has shown to be a valuable tool for bug species identification. To the best of our knowledge, the structure of legs has not been documented in detail so far and this is the first report of an open-closed rack system in legs of $C$. hemipterus. Because our sampling area was limited, further study is needed to determine the abundance species of bed bug in Iran.

\section{Declarations}




\section{Acknowledgment}

This study was supported by the research grant provided by the Shahid Chamran University of Ahvaz.

\section{Authors' contributions}

SB, IA, and FP contributed to overall study design. SB, IA and FP identifed study location, bugs collection, molecular and morphological identification. SB, IA, FP and CON analyzed the data. SB, SC, IA, FP and NCO drafted and revised the manuscript. All authors read and approved the fnal manuscript.

\section{Funding}

No funding.

\section{Availability of data and materials}

The datasets used and/or analyzed during the current study are available from the corresponding author upon request.

\section{Ethics approval and consent to participate}

Not applicable.

\section{Consent for pubication}

Not applicable.

\section{Completing interests}

The authors declare that they have no competing interests.

\section{References}

1. Zorrilla-Vaca A, Silva-Medina MM, Escandón Bedbugs, Cimex spp.: Their current world resurgence and healthcare impact. Asian Pac J Trop Dis 2015; 5: 342-352.

2. Mohammad Akhoundi, Remy Durand, Asad Mirzaei, Arezki Izri. Bed Bugs (Hemiptera, Cimicidae): Overview of Classification, Evolution and Dispersion. Int J Environ Res Publ Health 2020; 17: 4576.

3. Abd Rahim AH, Zahran Z, Ab Majid AH. Human skin reactions towards bites of tropical bed bug, Cimex hemipterus F. (Hemiptera: Cimicidae): A preliminary case study. Asian Pac J Trop Dis 2016; 6: 366-371.

4. Pritchard MJ, Hwang SW. Severe anemia from bedbugs. Can Med Assoc J 2009; 181: 287-288.

5. Potter MF. The perfect storm: an extension view on bed bugs. Am Entomol 2006; 52: 102-104.

6. Lilly DG, Latham SL, Webb CE, Doggett SL. Cuticle thickening in a pyrethroid-resistant strain of the common bed bug, Cimex lectularius(Hemiptera: Cimicidae). PloS One 2016; 11: e0153302.

7. Karunaratne S, Damayanthi B, Fareena M, Imbuldeniya V, Hemingway J. Insecticide resistance in the tropical bedbug Cimex hemipterus. Pestic Biochem Phys 2007; 88: 102-107.

8. Lai O, Ho D, Glick S, Jagdeo J. Bed bugs and possible transmission of human pathogens: a systematic review. Arch Dermatol Res 2016; 308: 531-538.

9. Reinhardt K, Siva-Jothy MT. Biology of the bed bugs (Cimicidae). Annu Rev Entomol 2007; 52: 351-374. 
10. Rosenfeld, J. A, Reeves D, Brugler MR, Narechania A, Simon S, Durrett R, Foox J, Shianna K, Schatz MC, Gandara J, Afshinnekoo E, Lam ET, Hastie AR, Chan S, Cao H, Saghbini M, Kentsis A, Planet PJ, Kholodovych V, Tessler M, Baker R, DeSalle R, Sorkin LN, Kolokotronis SO, Siddall ME, Amato G, Mason CE. Genome assembly and geospatial phylogenomics of the bed bug Cimex lectularius. Nat Commun 2016; 7:10164

11. Lai O, Ho D, Glick S, Jagdeo J. Bed bugs and possible transmission of human pathogens: a systematic review. Arch Dermatol Res 2016; 308(8): 531-538.

12. Zorrilla-Vaca A. Bedbugs and vector-borne diseases. Clin Infect Dis 2014; 59:1351-1352.

13. Narain RB, Lalithambika S, Kamble ST. Genetic variability and geographic diversity of the common bed bug (Hemiptera: Cimicidae) populations from the midwest using microsatellite markers. J Med Entomol 2015; 52(4): 566-572.

14. Kambhampati S, Smith P. PCR primers for the amplification of four insect mitochondrial gene fragments. Insect Mol Biol 1995; 4: 233-236.

15. Simon C, Frati F, Beckenbach A, Crespi B, Liu H, Flook P. Evolution, weighting, and phylogenetic utility of mitochondrial gene sequences and a compilation of conserved polymerase chain reaction primers. Ann Entomol Soc Am 1994; 87: 651-701.

16. Librado P, Rozas J. Dnasp v5: a software for comprehensive analysis of DNA polymorphism data. Bioinformatics 2009; 25: 1451-1452.

17. Tajima F. Evolutionary relationship of dna sequences in finite populations. Genetics, 1983; 105: 437-460.

18. Lynch M, Crease T. The analysis of population survey data on DNA sequence variation. Mol Biol Evol 1990; 7: 377-394.

19. Usinger RL. Monograph of Cimicidae (Hemiptera, Heteroptera). Thomas Say publications in entomology. Washington DC., Entomological Society of America. 1966; 585.

20. Carayon J. Traumatic insemination and the paragenital system. In: Usinger R.L, editor. Monograph of the Cimicidae (Hemiptera-Heteroptera)Entomological Society of America; College Park, 1966; 81-166.

21. Doggett SL, Miller DM, Lee CY. Advances in the biology and management of modern bed bugs, John Wiley \& Sons Ltd. 2018; PP: 472.

22. Kolb A, Needham GR, Neyman KM, High WA. Bedbugs. Dermatol Ther 2009; 22: 347-352.

23. Kress WJ, Erickson DL. Dna barcodes: genes, genomics, and bioinformatics. Proc Natl Acad Sci U S A 2008; 105 : 2761-2762.

24. Askari O, Farshbaf Pourabad R, Khaganinia S. Faunistic study of heteroptera of Zanjanroud region in Zanjan province of Iran. Mun Ent Zool 2009; 4: 560-563.

25. Haghi SFM, Behbodi M, Hajati H, Shafaroudi MM. Prevalence of bed bug (Cimexlectularius) in human settlement area of Bahnamir, Iran. Asian Pac J Trop Dis 2014; 4: 786-789.

26. Dehghani R, Hashemi A, Takhtfiroozeh SM, Chimehi E, Chimehi E. Bed bug (Cimexlectularis) outbreak: a crosssectional study in Polour, Iran. Iran J Dermatol 2016; 19: 16-20.

27. Ghahari H, Moulet P, Ostovan $H$. An annotated catalog of the Iranian cimicidae and largidae (Hemiptera: Heteroptera) and in memoriam carl walter schaefer (1934-2015). Zootaxa 2016; 4111: 194-200.

28. Sharififard M, Alizadeh I, Jahanifard E, Saki-Malehi A. Prevalence and spatial distribution of bed bug, Cimex lectularius, infestation in southwest of Iran: GIS approach. Iran J Arthropod-Borne Dis 2020; 14: $29-37$.

29. Szalanski AL, Tripodi AD, and Austin JW. Multiplex polymerase chain reaction diagnostics of bed bug (Hemiptera: Cimicidae). J Med Entomo/2011; 48: 937-940. 
30. Hosseini-Chegeni A, Gidiglo G, Khedri J. The first record of the tropical bed bug, Cimex hemipterus (Hemiptera: Cimicidae) from Iran. Iran J Anim Biosyst 2019; 15: 77-86.

31. Albert JT, Kozlov AS. Comparative aspects of hearing in vertebrates and insects with antennal ears. Curr Biol2016; 26: 1050-1061.

32. Krishnan A, Sane Antennal mechanosensors and their evolutionary antecedents. Adv Insect Physio/ 2015; 49: 5999.

33. Shields V, Hildebrand JG. Responses of a population of antennal olfactory receptor cells in the female moth manduca sexta to plant-associated volatile organic compounds. J Comp Physiol 2001; 186: 1135-1151.

34. Ruschioni S, Riolo P, Verdolini E, Peri E, Guarino S, Colazza S, Romani R, Isidoro N. Fine structure of antennal sensilla of Paysandisia archon and electrophysiological responses to volatile compounds associated with host palms. PLoS One 2015; 10(4):e0124607.

35. Singh RN, Singh K, Prakash S, Mendki M, Rao K. Sensory organs on the body parts of the bed-bug Cimex hemipterus fabricius (Hemiptera: Cimicidae) and the anatomy of its central nervous system. Int $J$ Insect Morphol Embryol 1996; 25: 183-204.

36. NowińskaA, Brożek Morphological study of the antennal sensilla in Gerromorpha (Insecta: Hemiptera: Heteroptera). Zoomorphol 2017; 136: 327-347.

37. Chen L, Fadamiro HY. Antennal sensilla of the decapitating phorid fly, Pseudacteon tricuspis (Diptera: Phoridae). Micron 2008: 39; 517-525.

38. Setzu MD, Poddighe S, Angioy AM. Sensilla on the antennal funiculus of the blow fly, protophormia terraenovae (Diptera: Calliphoridae). Micron 2011; 42: 471-477.

39. Liu XH, Zhang M, Shi JN, Li K, Zhang D. Ultrastructure of antennal sensilla of a parasitoid fly, Pales pavida meigen (Diptera: Tachinidae). Micron 2013; 54: 36-42.

40. Song LM, Wang XM, Huang JP, Zhu F, Jiang X, Zhang SG, Ban LP. Ultrastructure and morphology of antennal sensilla of the adult diving beetle Cybister japonicusPLoS One 2017; 12: e0174643.

41. Keil TA. Morphology and development of the peripheral olfactory organs. In: Hansson B.S. (eds) Insect Olfaction. Springer, Berlin, Heidelberg, 1999.

42. Olson JF, Moon RD, Kells SA, Mesce KA. Morphology, ultrastructure and functional role of antennal sensilla in offhost aggregation by the bed bug, Cimex lectularius. Arthropod Struct Dev 2014; 43: 117-122.

43. Seada MA, Hamza AM. Differential morphology of the sensory sensilla of antennae, palpi, foretarsi and ovipositor of adult Tribolium castaneum (Herbst) (Coleoptera: Tenebrionidae). Ann Agric Sci 2018; 63: 1-8.

44. Ren L, Shi J, Zhang Y, Luo Y. Antennal morphology and sensillar ultrastructure of Dastarcushelophoroides (fairmaire) (Coleoptera: Bothrideridae). Micron 2012; 43: 921-928.

45. Ruschioni S, Riolo P, Verdolini E, Peri E, Guarino S, Colazza S, Romani R, Isidoro N. Fine structure of antennal sensilla of Paysandisia archon and electrophysiological responses to volatile compounds associated with host palms. PLoS One 2015; 10: e0124607.

46. Yuan X, Zhang S, Zhang Z, Kong X, Wang H, Shen G, Zhang H. Antennal morphology and sensilla ultrastructure of the web-spinning sawfly Acantholyda posticalis Matsumura (Hymenoptera: Pamphiliidae). Micron 2013; 50: 20 28.

47. Yuvaraj JK, Andersson MN, Anderbrant O, Löfstedt C. Diversity of olfactory structures: A comparative study of antennal sensilla in Trichoptera and Lepidoptera. Micron 2018; 111: 9-18.

48. Wang Y, Li D, Liu Y, Li XJ, Cheng WN, Zhu-Salzman K. Morphology, ultrastructure and possible functions of antennal sensilla of sitodiplosis mosellana géhin (Diptera: Cecidomyiidae). J Insect Sci 2016; 16: 1-12. 
49. Kim DY, Billen J, Doggett SL, Lee CY. Differences in climbing ability of Cimex lectularius and Cimex hemipterus (Hemiptera: Cimicidae). J Econ Entomol 2017; 110: 1179-1186.

50. Baker GT, Goddard J. Structure and number of tibial brush setae making up the fossula spongiosa in bed bugs, Cimex lectularius (Hemiptera: Cimicidae). Proc Entomol Soc Wash 2018; 120: 251-254.

51. Seelinger G Tobin T. Sense organs. In "the American cockroach" (W. J. Bell and K. G Adiydi, eds.), Chapman \& Hall, London, 1981; pp: 217-245.

52. Kojima T. Developmental mechanism of the tarsus in insect legs. Curr Opin Insect Sci 2016; 19.

53. Cruz MS, Mateo MM. Scanning electron microscopy of legs of two species of sucking lice (Anoplura: Phthiraptera). Micron 2009; 40: 401-408.

\section{Tables}

Table 1.

Test of the homogeneity of substitution patterns between sequences. A Monte Carlo test (500 replicates) was used to estimate the $P$-values, which are shown in the table. $P$ values smaller than 0.05 are considered significant.

\begin{tabular}{|llllll|}
\hline & MT520974 & MT520975 & MT520976 & MT520977 & MT520978 \\
\hline MT520974 & - & - & - & - & - \\
\hline MT520975 & 1 & - & - & - & - \\
MT520976 & 1 & 1 & - & - & - \\
\hline MT520977 & 1 & 1 & 1 & - & - \\
\hline MT520978 & 1 & 1 & 1 & 0.43 & - \\
\hline
\end{tabular}

Table 2.

Estimates of evolutionary divergence between sequences. The number of base substitutions per site from between sequences is shown in the table. Analyses were conducted using the Tajima-Nei model. The rate variation among sites was modeled with a gamma distribution.

\begin{tabular}{|llllll|}
\hline & MT520974 & MT520975 & MT520976 & MT520977 & MT520978 \\
\hline MT520974 & - & - & - & - & - \\
\hline MT520975 & 0.03 & - & - & - & - \\
\hline MT520976 & 0.00 & 0.04 & - & - & - \\
\hline MT520977 & 0.06 & 0.05 & 0.06 & - & - \\
\hline MT520978 & 0.05 & 0.04 & 0.06 & 0.072 & - \\
\hline
\end{tabular}


Table 3.

Basic parameters of genetic and neutrality tests in five $C$. hemipterus samples collected from Iran ( $ð$ : Nucleotide diversity; Eta: Total number of mutations; Hap: Number of haplotypes; Hd: Haplotype diversity; Pi: The average number of nucleotide differences per site between two sequences; $\mathrm{G}+\mathrm{Cn}$ : $\mathrm{G}+\mathrm{C}$ content at the non-coding position).

\begin{tabular}{|c|c|c|c|c|c|c|c|c|c|c|c|}
\hline \multicolumn{10}{|c|}{ Diversity Parameters } & \multicolumn{2}{|c|}{ Neutrality test } \\
\hline $\begin{array}{l}\text { Net } \\
\text { sites }\end{array}$ & $\begin{array}{l}\text { Polymorphic } \\
\text { sites }\end{array}$ & $\begin{array}{l}\text { Singleton } \\
\text { variable } \\
\text { sites }\end{array}$ & $\begin{array}{l}\text { Parsimony } \\
\text { informative } \\
\text { sites }\end{array}$ & ð & Eta & Hap & $\mathrm{Hd}$ & $\mathrm{Pi}$ & $\mathrm{G}+\mathrm{Cn}$ & $\begin{array}{l}\text { Tajima' } \\
\text { D } \\
\text { p } ₫ 0.01\end{array}$ & $\begin{array}{l}\text { Fu' Fs } \\
\mathrm{p} \otimes 0.01\end{array}$ \\
\hline 323 & 28 & 16 & 14 & 0.57 & 30 & 5 & 1 & 0.04 & 0.26 & 0 & 0.13 \\
\hline
\end{tabular}

Table 4.

Conserved regions of the $16 \mathrm{~S}$ rDNA sequences of the C. hemipterus.

\begin{tabular}{|llll|}
\hline Region & Start-End & Homozygosity & P-value \\
\hline 1 & $1-42$ & 1 & 0.01 \\
2 & $44-92$ & 1 & 0 \\
\hline 3 & $261-333$ & 1 & 0 \\
\hline
\end{tabular}

\section{Figures}




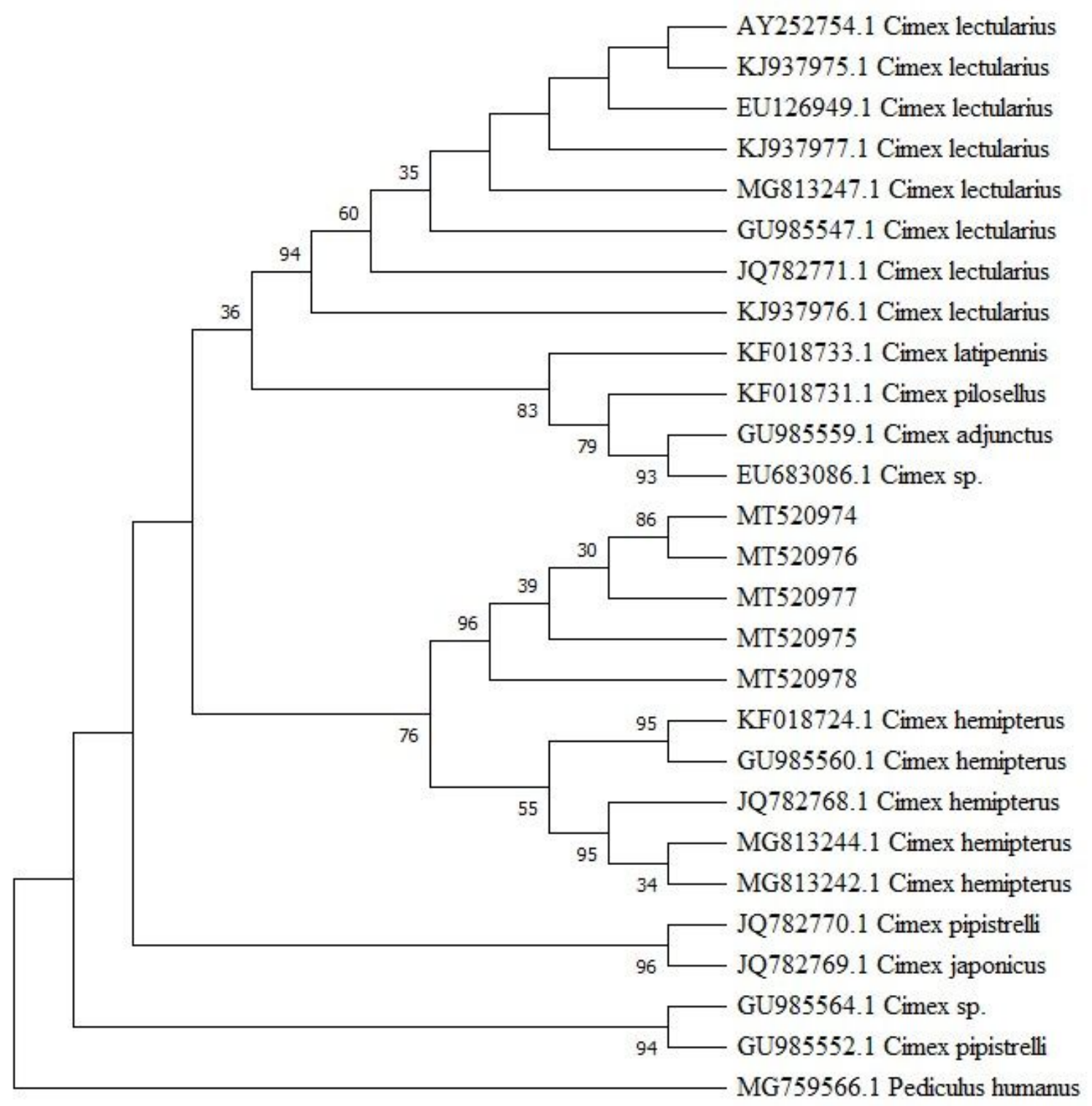

Figure 1

The phylogenetic tree was inferred by using the Maximum Likelihood method and Hasegawa-Kishino-Yano model. A discrete Gamma distribution was used to model evolutionary rate differences among sites. The reliability of an inferred tree was tested by 1000 bootstrap. This analysis involved five nucleotide sequences collected in this study (MT520974-MT520978), 21 nucleotide sequences from Genbank, and one outgroup (P. humanus capitis, AY139928.1). Evolutionary analyses were conducted in MEGA X. 


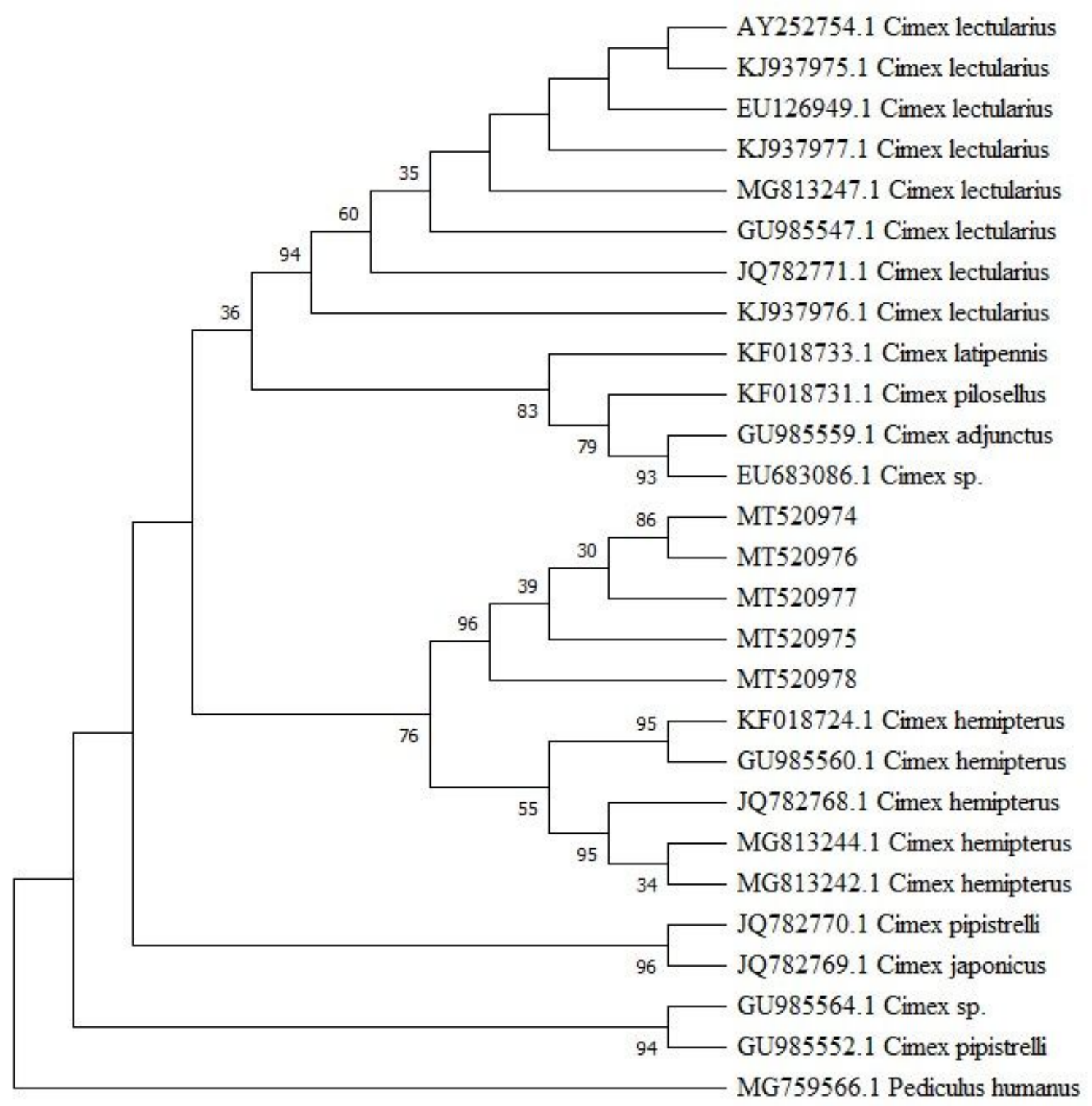

Figure 1

The phylogenetic tree was inferred by using the Maximum Likelihood method and Hasegawa-Kishino-Yano model. A discrete Gamma distribution was used to model evolutionary rate differences among sites. The reliability of an inferred tree was tested by 1000 bootstrap. This analysis involved five nucleotide sequences collected in this study (MT520974-MT520978), 21 nucleotide sequences from Genbank, and one outgroup (P. humanus capitis, AY139928.1). Evolutionary analyses were conducted in MEGA X. 


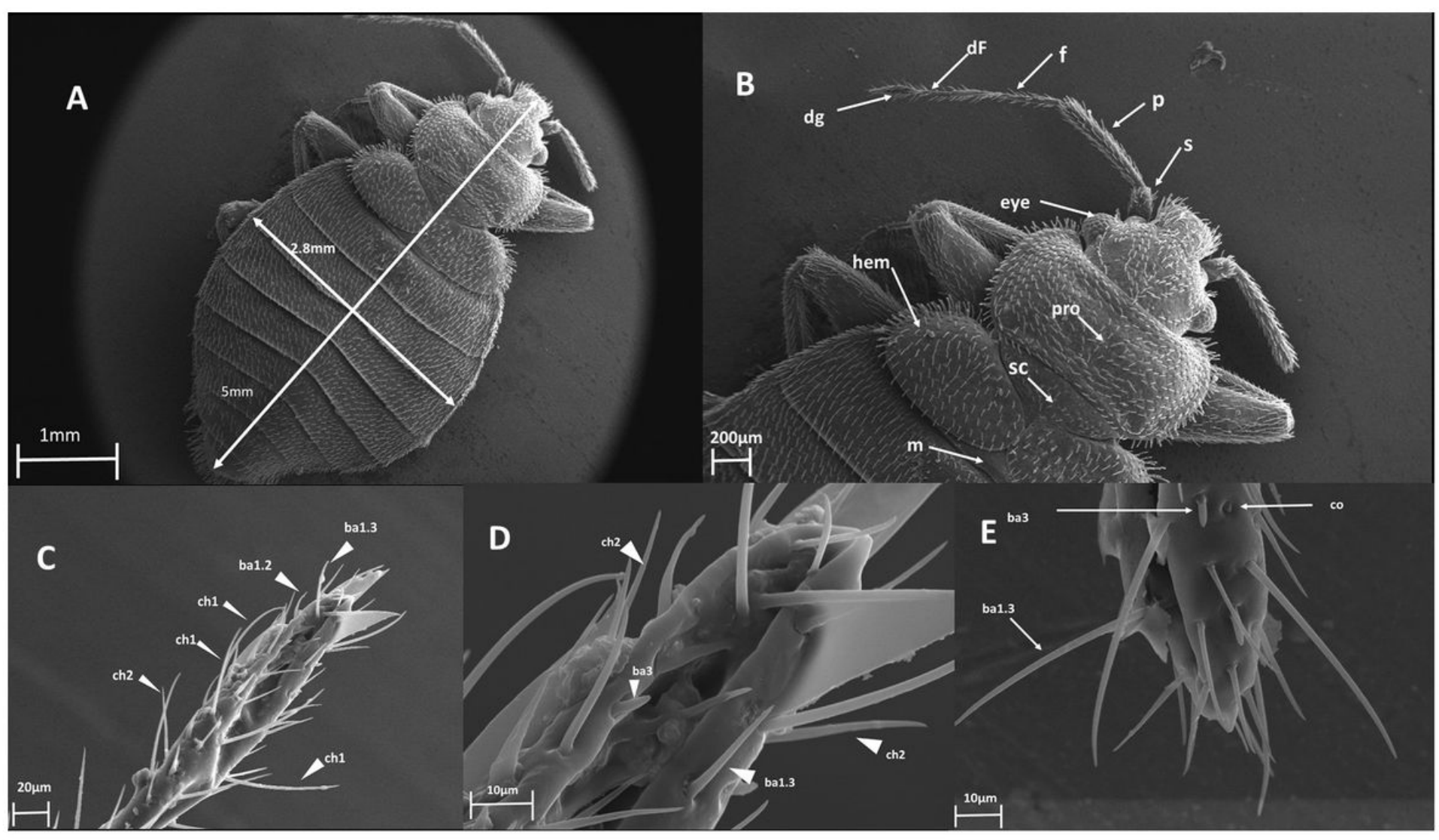

\section{Figure 2}

Shows the antenna and its sensilla. Scape (S), pedicel (p), flagellum (f), and distal flagellum (df), deep groove (dg), pronotum (pro), hemelytral pads (hem), scutellum (sc), metathorax (m). 


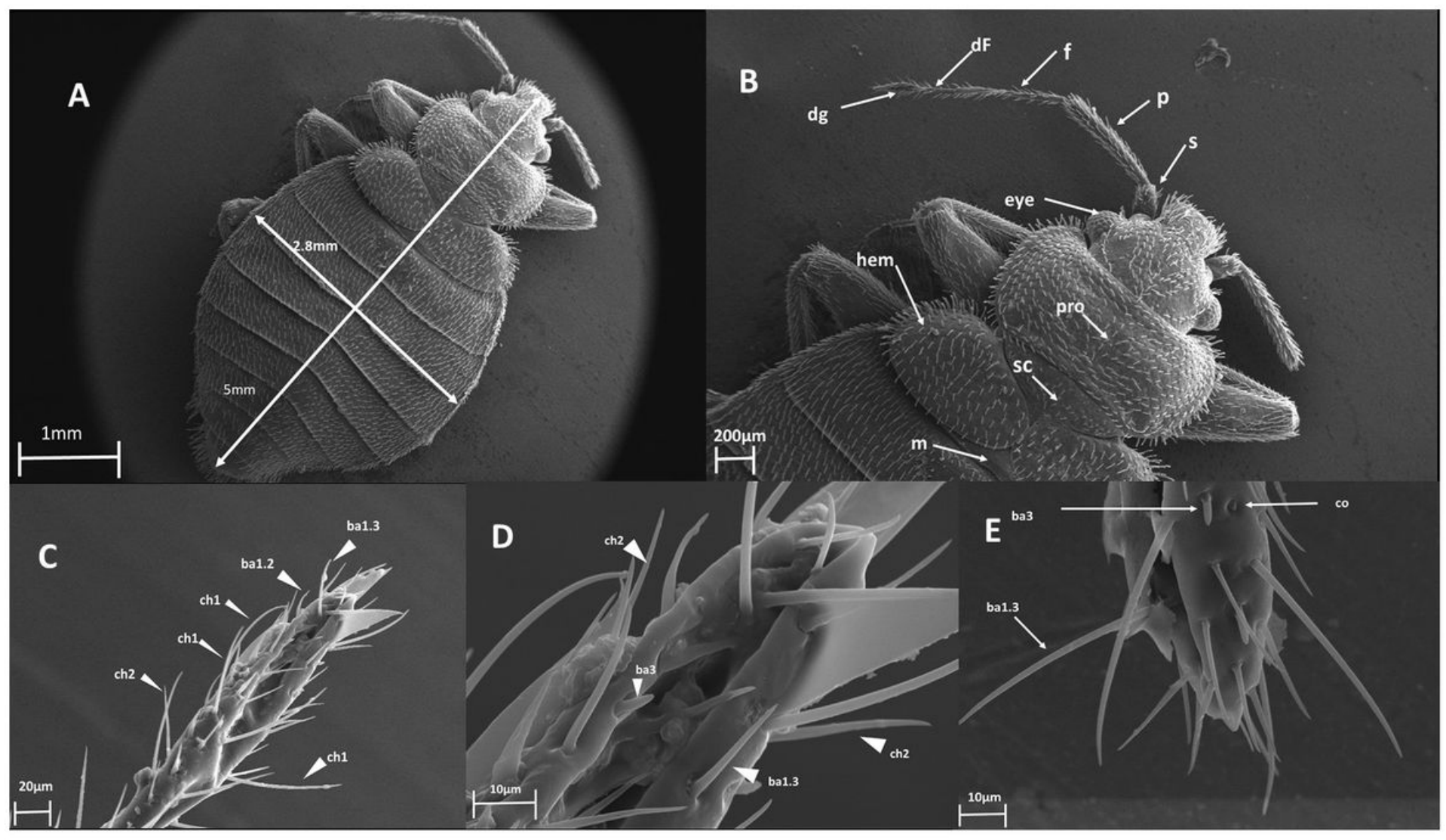

\section{Figure 2}

Shows the antenna and its sensilla. Scape (S), pedicel (p), flagellum (f), and distal flagellum (df), deep groove (dg), pronotum (pro), hemelytral pads (hem), scutellum (sc), metathorax (m).

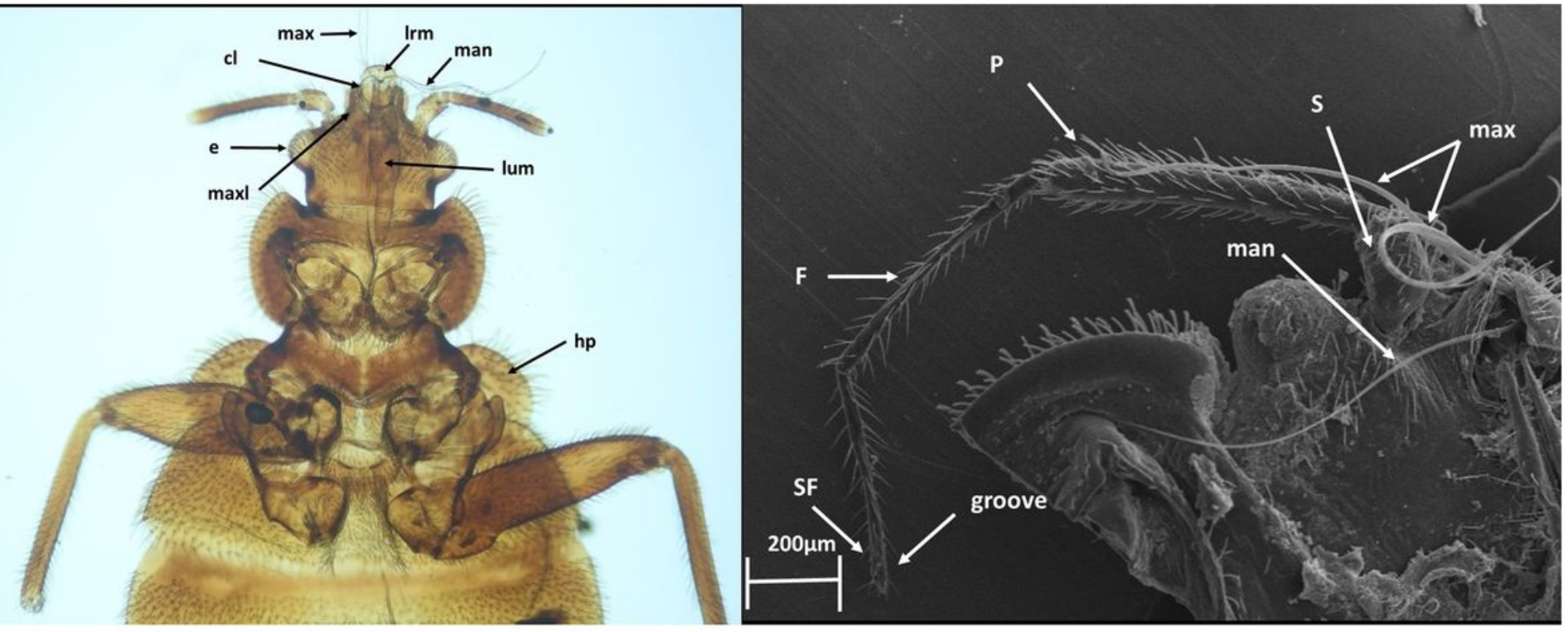

\section{Figure 3}

Shows the mouthparts of C. hemipterus. Maxilla (max), mandibles (man), labrum (Irm), labium (lum), rostrum (ro), hemelytral pad (hp), clypeus (cl), Eye (e), maxillary lobe (maxl). 


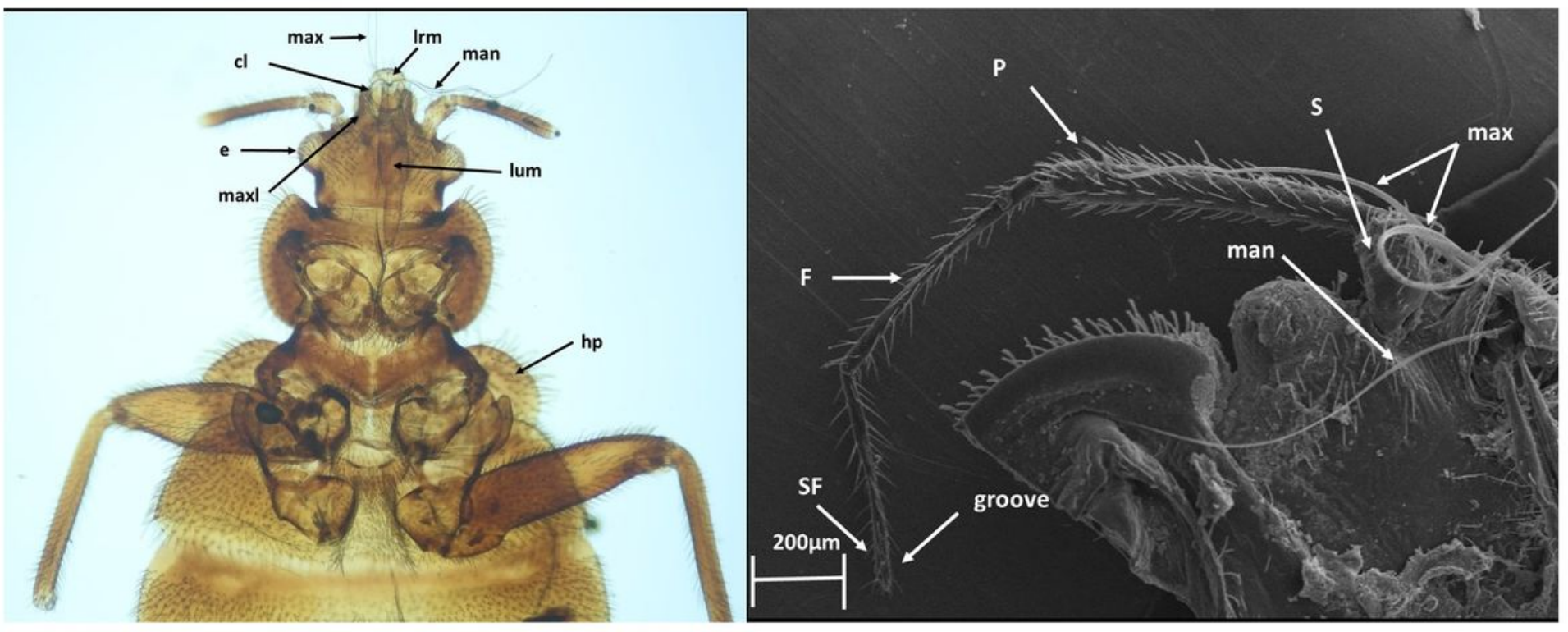

\section{Figure 3}

Shows the mouthparts of C. hemipterus. Maxilla (max), mandibles (man), labrum (Irm), labium (lum), rostrum (ro), hemelytral pad (hp), clypeus (cl), Eye (e), maxillary lobe (maxl).

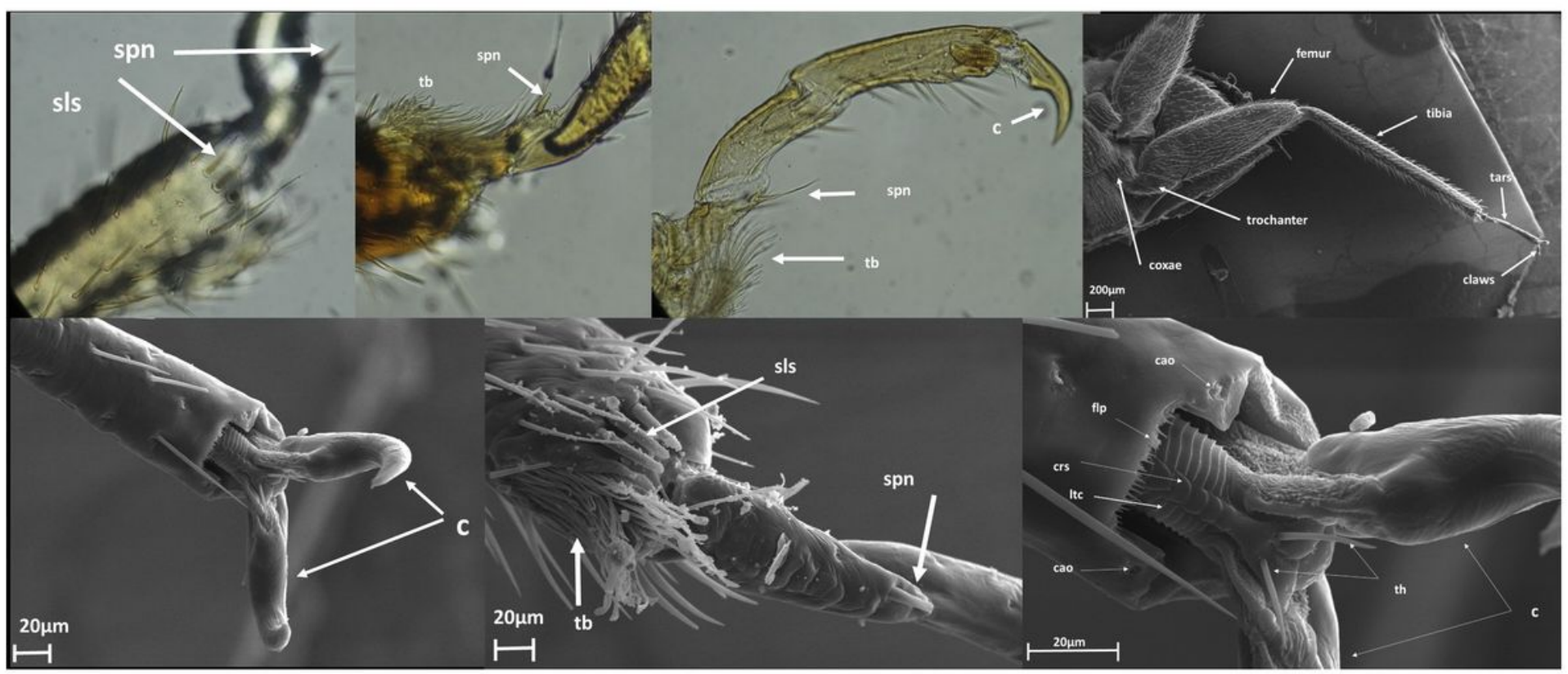

\section{Figure 4}

Shows several structures in pretarsus. Open- closed system is shown in C. hemipterus legs. Claw (c), tibial brush (tb), spine of the thumb (spn), stout long spines (sls), coupled finger-like process(flp), rack- system (crs), campaniform organ (cao), tactile hair (th), lateral telescopic columns (Itc). 


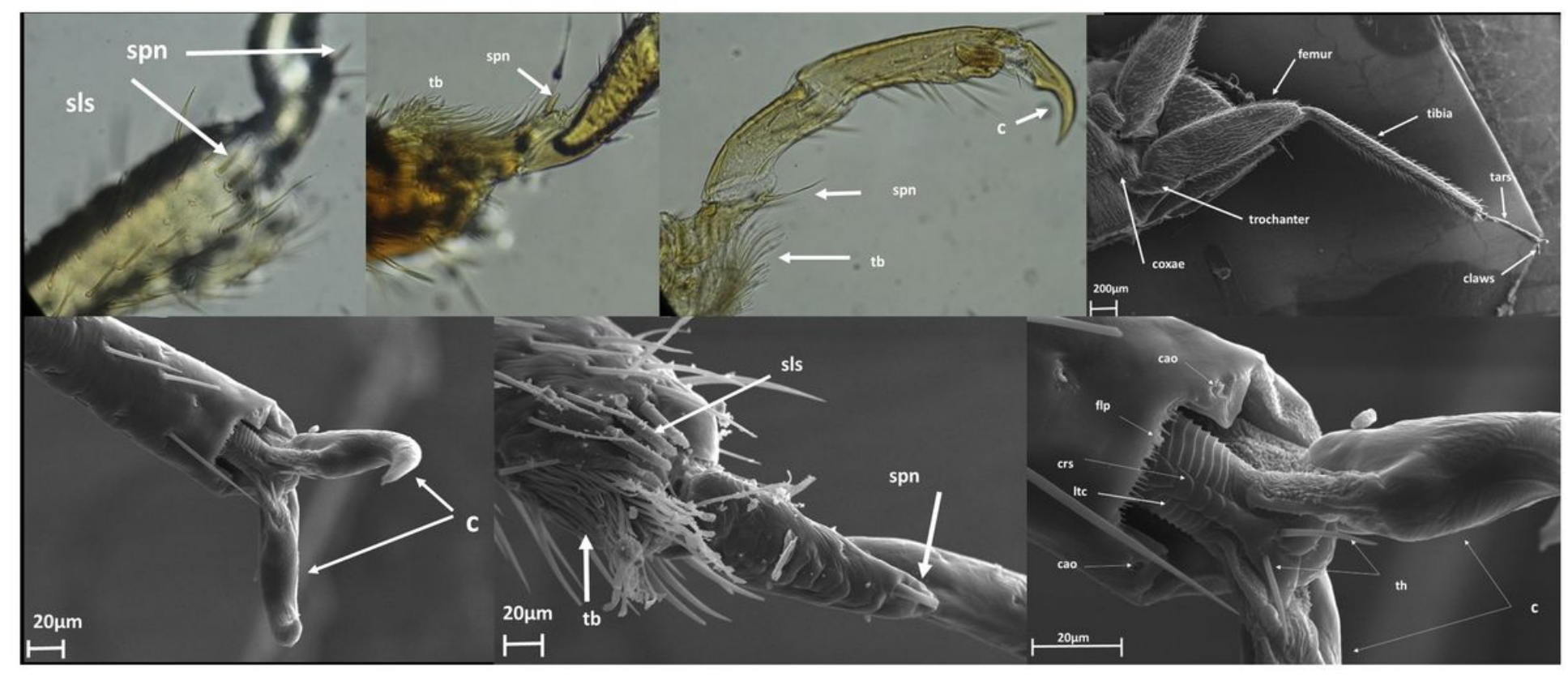

\section{Figure 4}

Shows several structures in pretarsus. Open- closed system is shown in C. hemipterus legs. Claw (c), tibial brush (tb), spine of the thumb (spn), stout long spines (sls), coupled finger-like process(flp), rack- system (crs), campaniform organ (cao), tactile hair (th), lateral telescopic columns (Itc). 


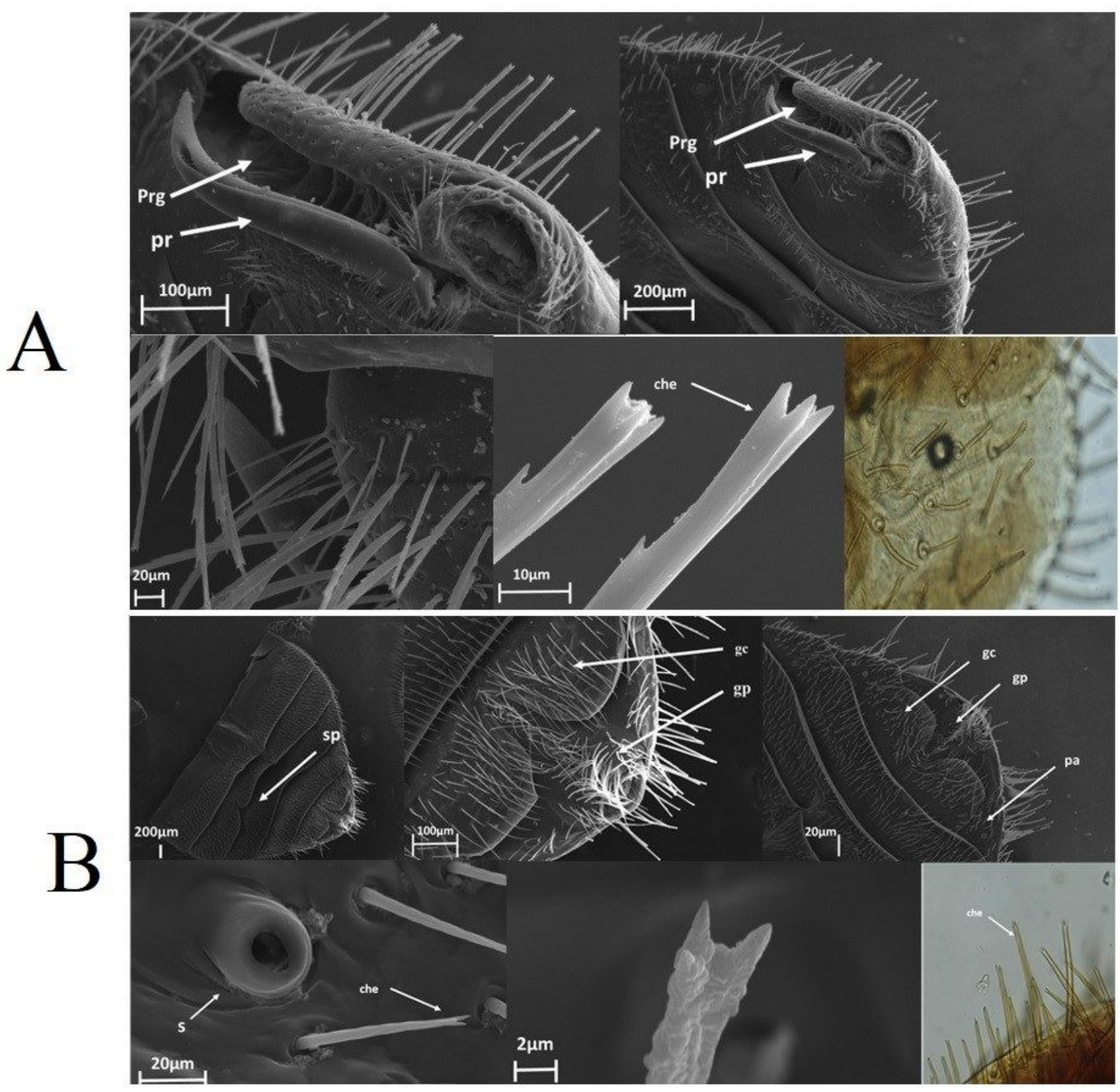

\section{Figure 5}

A: shows the paramere (pr), paramere groove (prg), and male bugs chaetae with four teeth in the tip (che). B: shows the spermalege (sp), gonocoxite (gc), gonapophysis (gp), paratergite (pa), spiracle (s), and female bugs chaetae with three teeth in the tip (che). 


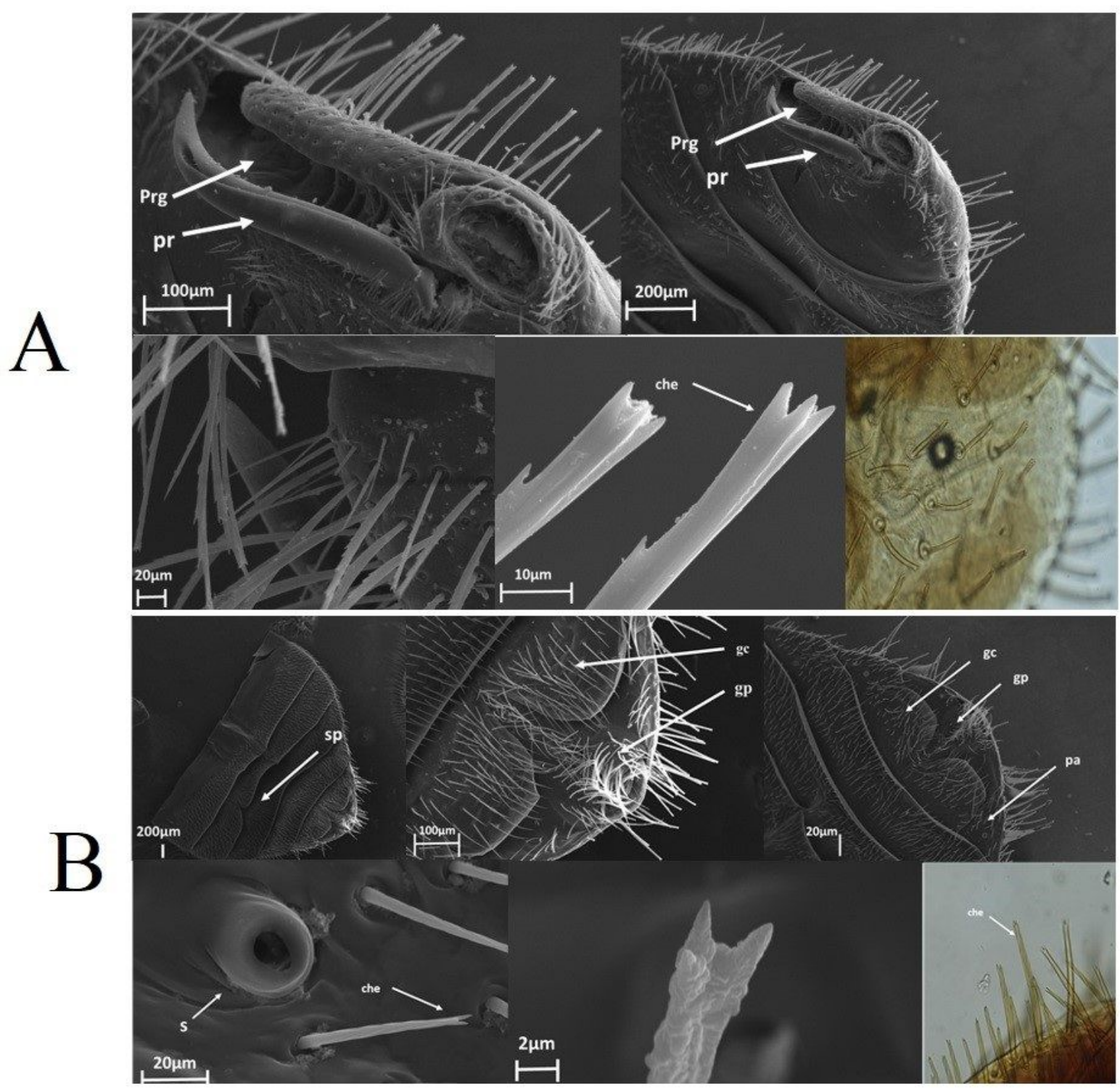

\section{Figure 5}

A: shows the paramere (pr), paramere groove (prg), and male bugs chaetae with four teeth in the tip (che). B: shows the spermalege (sp), gonocoxite (gc), gonapophysis (gp), paratergite (pa), spiracle (s), and female bugs chaetae with three teeth in the tip (che).

\section{Supplementary Files}

This is a list of supplementary files associated with this preprint. Click to download.

- GraphicAbstract.jpg

- GraphicAbstract.jpg 